\title{
A novel metabolomic approach used for the comparison of Staphylococcus aureus planktonic cells and biofilm samples
}

\author{
Laurence H. Stipetic ${ }^{1,2}$ - Matthew J. Dalby ${ }^{3}$ Robert L. Davies ${ }^{1}$ - Fraser R. Morton ${ }^{2}$. \\ Gordon Ramage $^{4} \cdot$ Karl E. V. Burgess ${ }^{1,2}$
}

Received: 16 October 2015/Accepted: 16 January 2016/Published online: 8 March 2016

(c) The Author(s) 2016. This article is published with open access at Springerlink.com

\begin{abstract}
Introduction Bacterial cell characteristics change significantly during differentiation between planktonic and biofilm states. While established methods exist to detect and identify transcriptional and proteomic changes, metabolic fluctuations that distinguish these developmental stages have been less amenable to investigation.

Objectives The objectives of the study were to develop a robust reproducible sample preparation methodology for high throughput biofilm analysis and to determine differences between Staphylococcus aureus in planktonic and biofilm states.

Methods The method uses bead beating in a chloroform/ methanol/water extraction solvent to both disrupt cells and quench metabolism. Verification of the method was performed using liquid-chromatography-mass spectrometry. Raw mass-spectrometry data was analysed using an in-house bioinformatics pipe-line incorporating XCMS, MzMatch
\end{abstract}

Electronic supplementary material The online version of this article (doi:10.1007/s11306-016-1002-0) contains supplementary material, which is available to authorized users.

Karl E. V. Burgess

karl.burgess@glasgow.ac.uk

1 Institute of Infection, Immunity and Inflammation, College of Medical, Veterinary and Life Sciences, The University of Glasgow, Glasgow, UK

2 Glasgow Polyomics, Wolfson Wohl Cancer Research Centre, The University of Glasgow, Garscube Estate, Bearsden, Scotland G61 1QH, UK

3 Institute of Molecular Cell and Systems Biology, The University of Glasgow, Glasgow, UK

4 Infection and Immunity Research Group, Glasgow Dental School, School of Medicine, College of Medical, Veterinary and Life Sciences, The University of Glasgow, Glasgow, UK and in-house R-scripts, with identifications matched to internal standards and metabolite data-base entries.

Results We have demonstrated a novel mechanical bead beating method that has been optimised for the extraction of the metabolome from cells of a clinical Staphylococcus aureus strain existing in a planktonic or biofilm state. This high-throughput method is fast and reproducible, allowing for direct comparison between different bacterial growth states. Significant changes in arginine biosynthesis were identified between the two cell populations.

Conclusions The method described herein represents a valuable tool in studying microbial biochemistry at a molecular level. While the methodology is generally applicable to the lysis and extraction of metabolites from Gram positive bacteria, it is particularly applicable to biofilms. Bacteria that exist as a biofilm are shown to be highly distinct metabolically from their 'free living' counterparts, thus highlighting the need to study microbes in different growth states. Metabolomics can successfully distinguish between a planktonic and biofilm growth state. Importantly, this study design, incorporating metabolomics, could be optimised for studying the effects of antimicrobials and drug modes of action, potentially providing explanations and mechanisms of antibiotic resistance and to help devise new antimicrobials.

Keywords Metabolomics - Metabolite extraction · Biofilms $\cdot S$. aureus

\section{Introduction}

Metabolomics aims to measure comprehensively the metabolic profile of a system (Fiehn 2002). The term metabolomics was first coined in 1998 (Oliver et al. 1998), 
although the concept of 'metabolic profiling' can be dated to the 1970s (Horning and Horning 1971). Metabolomics provides a 'snap-shot' of cell metabolism and can highlight discrete changes in metabolic pathways and the abundance of biological small molecule metabolite intermediates. Using untargeted metabolomics, chemical effects across the gamut of metabolic processes can be studied providing greater understanding of the biological responses from single cells to the complex system/organism level (Nicholson and Lindon 2008).

Metabolomic techniques have been applied to bacterial cells (Tang 2011; Liebeke et al. 2012), although not without significant hurdles. Current microbial extraction and metabolic quenching methods have a number of associated problems (de Koning and van Dam 1992; Bolten et al. 2007). Often, metabolite degradation or inadequate quenching of metabolism during extraction, media contamination, or failure to lyse the bacterial cell, can lead to artefactual and/or variable results (Bolten et al. 2007). In addition, many of the methods developed are primarily for studies of Gram-negative bacteria, which are poorly suited to Gram-positive bacteria because of their dense peptidoglycan cell wall (Maharjan and Ferenci 2003). Many bacteria also have the capacity to form biofilms on a range of biological and abiotic substrates (O'Toole et al. 2000). Here, an extracellular matrix (ECM) encases bacteria, altering phenotype, biochemistry and transcriptome in comparison to free floating, planktonic, cells. Biofilms also alter the ability to extract metabolites from bacteria as well as making them recalcitrant to antimicrobial chemotherapy (Ramage et al. 2003; Drenkard and Ausubel 2002). Moreover, the transition from planktonic growth to biofilm formation can influence the metabolic profile of the bacteria (Gjersing et al. 2007). These factors make the metabolic analysis of clinically important pathogens problematic.

Metabolomics offers novel methods to study fundamental processes involved in microbial biofilm formation, their response to antimicrobial chemotherapy, and may also lead to the identification of novel biomarkers that could improve clinical diagnostics. To date, limited investigations have been performed on metabolomics of biofilms using nuclear magnetic resonance (NMR) spectrometry to study molecular differences between planktonic cells and biofilms (Zhang and Powers 2012; Ammons et al. 2014; Gjersing et al. 2007).

This paper aims to describe a novel method applicable to study biofilm metabolism in the model Gram-positive pathogen Staphylococcus aureus. Here, we report for the first time an optimised method for the extraction of the metabolome directly from a biofilm in parallel to cells living in suspension. Through liquid-chromatography-mass-spectrometry (LC-MS) metabolomics we demonstrate that the method is highly reproducible, further showing significant differences in metabolism between planktonic and biofilm cells grown under identical conditions. We focus on differences in arginine biosynthesis between the two growth states, thus providing insights into the biological properties of this important nosocomial pathogen.

\section{Materials and methods}

All reagents and chemicals were purchased from Fisher Scientific, Loughborough, UK, at HPLC grade, unless stated otherwise.

\subsection{Bacteria culture}

A clinical $S$. aureus strain, LHSKBClinical, (Stipetic et al. 2015), was used throughout this study. This was cultured on Brain Heart Infusion (BHI) agar plates or in BHI broth media (Oxoid, Basingstoke, UK). For all experiments, single colonies were taken from a plate and inoculated into liquid media. Cultures were grown overnight at $37{ }^{\circ} \mathrm{C}$ to stationary phase growth, which was used for subsequent planktonic and biofilm studies. Bacteria were grown under aerobic conditions.

\subsection{Planktonic cell preparation}

For planktonic cell preparation, $200 \mu \mathrm{L}$ of the stationary phase overnight culture were added to wells of a 96-well microtitre plate (Corning Incorporated, New York, USA), centrifuged at $1900 \times g$ at $4{ }^{\circ} \mathrm{C}$ for $3 \mathrm{~min}$, and the spent media removed. Subsequently, $200 \mu \mathrm{l}$ of $10 \mathrm{mM}$ ammonium bicarbonate (Sigma-Aldrich, Dorset, UK) was added, the pellet resuspended, cells were washed by centrifugation at $1900 \times \mathrm{g}$ at $4{ }^{\circ} \mathrm{C}$ for $3 \mathrm{~min}$, and the supernatant was removed, providing a pellet for bead beating as described below.

\subsection{Biofilm cell preparation}

Biofilms were prepared by adding standardised $S$. aureus culture at $1 \times 10^{8}$ cells $/ \mathrm{ml}$ in BHI into a 96-well microtitre plate, which was then statically incubated for $18 \mathrm{~h}$ at $37{ }^{\circ} \mathrm{C}$ under aerobic conditions. Following incubation, the supernatant was removed and the biofilms were washed in sterile $10 \mathrm{mM}$ ammonium bicarbonate.

\subsection{Biofilm SEM}

Scanning electron microscopy (SEM) was performed on parallel samples of $S$. aureus biofilms cultivated directly onto Thermanox ${ }^{\mathrm{TM}}$ cover-slips (Nunc, Roskilde, 
Denmark), processed and imaged as previously described by Erlandsen et al. (2004).

\subsection{Metabolite extraction using bead beating}

Metabolites were extracted directly within the wells of a microtitre plate by adding $200 \mu \mathrm{l}$ of $0.1 \mathrm{~mm}$ acid washed, glass beads (Sigma-Aldrich) and ice cold $\left(-20{ }^{\circ} \mathrm{C}\right)$ solvent solution $(1 \mathrm{~g}$ of beads to $1 \mathrm{ml}$ of chloroform: methanol: $\mathrm{ddH}_{2} \mathrm{O}$ [ratio of $\left.1: 3: 1\right]$ ).

A 96-well microtitre plate was used, primarily so that biofilms could be extracted in situ and so that 24 independent replicates could be performed for each condition, including media only negative controls. The plate was then sealed with a 96-well Cap Mat (Greiner Bio-One, Stonehouse, UK) and bead beaten on a cell disrupter (Disrupter Genie $^{\circledR}$ ) (Scientific industries, Inc., New York, USA), operating at a speed of $3000 \mathrm{RPM}$, at $4{ }^{\circ} \mathrm{C}$ continuously for $10 \mathrm{~min}$. The plates were then centrifuged at $1900 \times g$ at $4{ }^{\circ} \mathrm{C}$ for $10 \mathrm{~min}$, to remove beads and cell debris. The supernatant was then removed and stored at $-80{ }^{\circ} \mathrm{C}$ until MS analysis. Extractions and metabolomics results represent one experiment with 24 replicates intended to demonstrate the utility of the method.

\subsection{Comparative methods for metabolite extractions}

Two additional, previously published methods for Gram positive bacterial cell lysis (Soga et al. 2002; Soga et al. 2003; Takahashi et al. 2010), were compared to the bead beating method. Modifications to the published methods were made, and are described in detail in Online resource 1 , but briefly, extraction solvents were altered to a chloroform/methanol/water 1:3:1 $\mathrm{mix}$ and water/PBS washes were replaced with $10 \mathrm{mM}$ ammonium bicarbonate to ensure differences between the methods were due to lysis rather than extraction solvent. Sonication was altered from $30 \mathrm{~s}$ with a sonic probe to $30 \mathrm{~min}$ in a sonic bath to minimise heating and provide compatibility with microtitre plate extractions. Each extraction method was performed in triplicate.

\subsection{Metabolomic workflow, data acquirement and analysis}

Samples were analysed by hydrophilic interaction liquid chromatography (HILIC) -mass spectrometry (LC-MS) (UltiMate 3000 RSLC (Thermo Fisher, San Jose, California, USA) using a 150 x $4.6 \mathrm{~mm}$ ZIC-pHILIC column (Merck SeQuant, Umea, Sweden) running at $300 \mu \mathrm{l} / \mathrm{min}$ and Orbitrap Exactive (Thermo Fisher) detection. Mass spectrometer parameters were: 50,000 resolving power in positive/negative switching mode. Electrospray ionisation (ESI) voltage was $4.5 \mathrm{kV}$ in positive and $3 \mathrm{kV}$ in negative modes. Buffers consisted of A: $20 \mathrm{mM}$ ammonium carbonate in $\mathrm{H}_{2} \mathrm{O}$ and $\mathrm{B}$ : Merck SeQuant: acetonitrile. The gradient ran from $20 \% \mathrm{~A}$ : $80 \%$ B to $80 \%$ A: $20 \%$ B in 15 min, followed by a wash at $95 \%$ A: $5 \%$ B for 3 min, and equilibration at $20 \%$ A: $80 \%$ $\mathrm{B}$ for $5 \mathrm{~min}$. Raw mass spectrometry data was processed using our standard pipeline, consisting of XCMS (Smith et al. 2006) (for peak picking), MzMatch (Scheltema et al. 2011) (for filtering and grouping) and in-house R-scripts (for further filtering, post-processing and identification). Peaks were visualised using PeakML Viewer (Scheltema et al. 2011). Core metabolite identifications were validated against a panel of unambiguous authentic pure standards using accurate mass and retention time $\left(\mathrm{R}_{\mathrm{t}}\right)$ and therefore could be classified using the alphanumeric metabolite coding scheme as $\operatorname{HRMS}_{\mathrm{a}}^{1} \mathrm{R}_{\mathrm{ta}}$ as described by Sumner et al. (2014). Additional putative identifications were assigned by accurate mass along with a $R_{t}$ prediction algorithm (Creek et al. 2011) and therefore could be classified as $\operatorname{HRMS}_{\mathrm{PL}}^{1}$ (Sumner et al. 2014). Quantile normalisation was carried out across the data, normalising both data sets (Bolstad et al. 2003). Once identified and filtered, detected peak intensities were logged (base 2) and quantitation was performed on sets of biological replicates by applying differential statistics to generate $\mathrm{P}$ values. Metabolites with apparently different levels were assessed using Bayes moderated t-tests (Smyth 2004). Benjamini and Hochberg false discovery rate adjustment for multiple testing was applied (Hochberg and Benjamini 1990) and the resulting data was used to query KEGG (Kyoto Encyclopaedia of Genes and Genomes data base) for pathway analysis (Kanehisa and Goto 2000). Statistics, including principal component analysis (PCA), were performed and presented using R, employing appropriate standard R libraries and Microsoft Excel. ChemDraw Std version 14.0 was used to draw metabolic pathways and GraphPad Prism 4 used to create figures. Comparative metabolomic analysis of extraction methods was further processed using the IDEOM software (Creek et al. 2012), without normalisation applied.

\section{Results}

\subsection{Liquid Chromatography-Mass Spectrometry metabolomics for $S$. aureus planktonic cell and biofilm analysis following sample bead- beating and metabolome extraction}

Static biofilms were grown using BHI, as an optimal medium based on a study of $S$. aureus biofilm formation in 1000 clinical isolates (Smith et al. 2008). SEM imaging (Fig. 1) provided qualitative data illustrating that the 

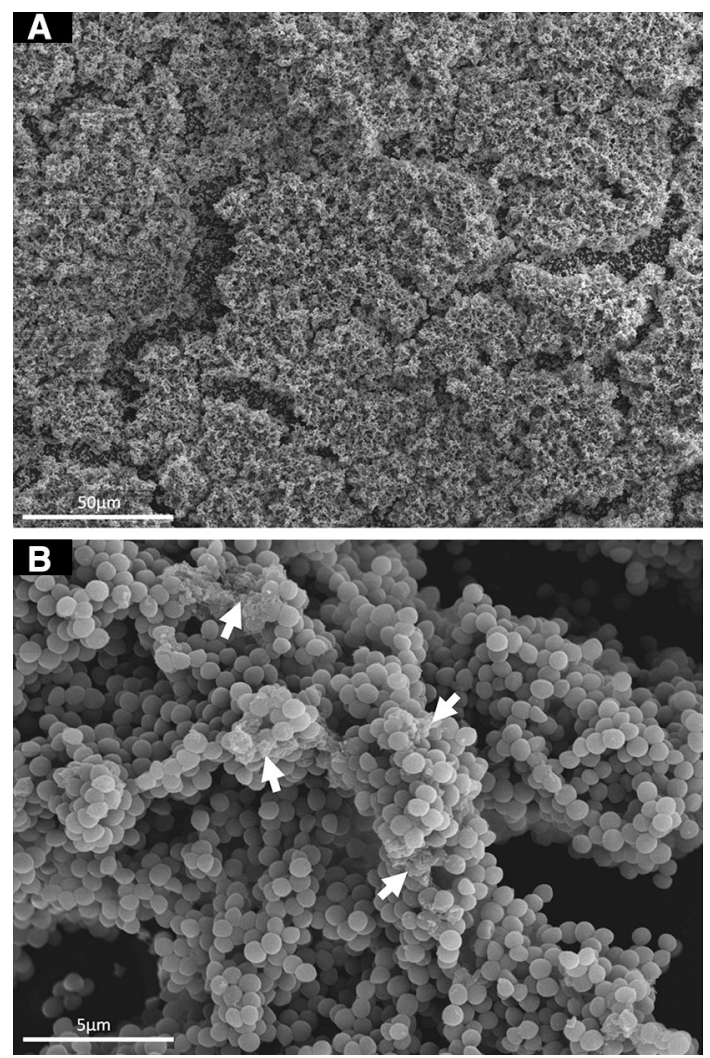

Fig. 1 Scanning Electron Microscopy images of a clinical S. aureus strain biofilm, cultivated in BHI directly on Thermanox ${ }^{\mathrm{TM}}$ cover-slips for $18-20$ h. a $500 \times$ magnification, b $5000 \times$ magnification. Arrows highlight areas of extracellular matrix

S. aureus clinically-derived strain used herein forms a biofilm. Figure 1 shows key characteristics of biofilm formation: cell clustering of cells attached and colonised to the surface of the cover-slip, and ECM production (O'Toole et al. 2000; Periasamy et al. 2012).

Once bacterial culture and biofilm cultivation had been established, an extraction platform was developed that would enable both planktonic and biofilm cells to be extracted simultaneously using a mechanical bead beating method.

A mechanical cell lysis method was optimised using $0.1 \mathrm{~mm}$ beads, as recommended for bacterial species by the cell disrupter manufacturer's guidelines (Scientific industries 2014). Initial bead beating studies demonstrated that 10 min induced $>95 \%$ cell death (data not shown).

Greiner Bio-One Cap mats were found to be optimal for sealing plates during lysis, while the use of plastic-paraffin film resulted in sample leakage and cross contamination between samples. Ammonium bicarbonate was used for all wash stages due to its LC-MS compatibility and moderate pH buffering (Cassou et al. 2014; Faijes et al. 2007; Hedges et al. 2013). Extracting the metabolites at $4{ }^{\circ} \mathrm{C}$ in chloroform/methanol/water slowed (Laidler 1984) and quenched (t'Kindt and 2010) cellular metabolic activity during extraction.

A significant consideration for metabolomics is the amount of material (metabolite extraction) required for an effective analysis. Using the LC-MS set-up utilised here, a minimum sample volume of $10 \mu \mathrm{l}$ of metabolite extraction with a minimum metabolite concentration dependent on the molecule studied, but detection limits generally in the micromolar range for small molecules (Gross 2011), were required for effective analysis. Sufficient material for detection and quantitation of hundreds of metabolites was obtained from a biofilm covering the well base of a 96-well microtitre plate.

\subsection{Comparison with alternative lysis methods}

The extraction process described herein was compared to the methods described by Soga et al. $(2002,2003)$ and Takahashi et al. (Takahashi et al. 2010). No viable bacteria were found after analysis of the pellet using any of the described methods (data not shown). Total useable signal of the metabolites from each method showed no significant difference between the lysis methods $(t$ test $\mathrm{P}$ values of 0.12 - bead beating vs. filtration, 0.10 - bead beating vs. sonication for positive mode and 0.15 -bead beating vs. filtration, 0.09 bead beating vs. sonication for negative mode data) (see Online resource 2). Further analysis of the extractions performed using the filtration method, however, demonstrated a general decrease in the intensities of well characterised metabolites. Of 84 'identified' metabolites, 31 are significantly lower in intensity with only 3 significantly higher, while the bead beating and sonication methods gave similar results overall (see Online resource 2).

\subsection{Liquid-chromatography-mass spectrometry (LC-MS) data analysis}

Data analysis of cell extractions derived from planktonic shaking cultures compared to static biofilm extractions yielded 530 significantly changing peaks with an adjusted $\mathrm{P}$ value of $<0.05$ (null hypothesis: there is no difference between planktonic cell and biofilm extractions, reject null hypothesis). Of these, 151 metabolites demonstrated a significant up-regulation in expression in planktonic cells compared to biofilms, with $\log _{2}$ fold changes of $\geq 1$. Conversely, 177 were found to be significantly up-regulated in biofilms as compared to planktonic cells with $\log _{2}$ fold changes of $\geq 1$. Metabolites were identified by matching mass and $R_{t}$ to internal standards or annotated by mass-matching to a database entry. Data analyses, including raw peak data, metabolite identification, planktonic compared to biofilm samples analysis, and $R_{t}$ errors are shown in Online Resource 3. 
Table 1 Arginine Biosynthesis metabolites identified from planktonic and biofilm data sets

\begin{tabular}{llllllll}
\hline Peak number & Metabolite & Elemental formula & KEGG ID $^{\mathrm{a}}$ & Metabolite code $^{\mathrm{b}}$ & Mass [M-H] $^{(\mathrm{Da})}$ & $\mathrm{R}_{\mathrm{t}}^{\mathrm{c}}(\mathrm{sec})$ & Log $_{2}$ fold change \\
\hline 2782 & Aspartate & $\mathrm{C}_{4} \mathrm{H}_{7} \mathrm{NO}_{4}$ & $\mathrm{C} 00049$ & $\mathrm{HRMS}_{\mathrm{PL}}^{1}$ & 132.0302 & 749.1 & 1.577 \\
2873 & Glutamate & $\mathrm{C}_{5} \mathrm{H}_{9} \mathrm{NO}_{4}$ & $\mathrm{C} 00025$ & $\mathrm{HRMS}_{\mathrm{a}}^{1} \mathrm{R}_{\mathrm{ta}}$ & 146.0458 & 725.7 & 1.0159 \\
2981 & Glutamine & $\mathrm{C}_{5} \mathrm{H}_{10} \mathrm{~N}_{2} \mathrm{O}_{3}$ & $\mathrm{C} 00064$ & $\mathrm{HRMS}_{\mathrm{a}}^{1} \mathrm{R}_{\mathrm{ta}}$ & 145.0618 & 781.3 & $\mathrm{n} / \mathrm{a}^{(\mathrm{e})}$ \\
3171 & Citrulline & $\mathrm{C}_{6} \mathrm{H}_{13} \mathrm{~N}_{3} \mathrm{O}_{3}$ & $\mathrm{C} 00327$ & $\mathrm{HRMS}_{\mathrm{a}}^{1} \mathrm{R}_{\mathrm{ta}}$ & 174.0884 & 813.1 & 3.5413 \\
3252 & N-Acetyl-L- & $\mathrm{C}_{7} \mathrm{H}_{11} \mathrm{NO}_{5}$ & $\mathrm{C} 00624$ & $\mathrm{HRMS}_{\mathrm{a}}^{1} \mathrm{R}_{\mathrm{ta}}$ & 188.0564 & 691.1 & 4.3654 \\
3505 & glutamate & & & & & \\
3608 & L-Arginosuccinate & $\mathrm{C}_{10} \mathrm{H}_{18} \mathrm{~N}_{4} \mathrm{O}_{6}$ & $\mathrm{C} 03406$ & $\mathrm{HRMS}_{\mathrm{PL}}^{1}$ & 289.1156 & 800.5 & $\mathrm{n} / \mathrm{a}^{\mathrm{f}}$ \\
3636 & N-Acetyl-L-citrulline & $\mathrm{C}_{8} \mathrm{H}_{15} \mathrm{~N}_{3} \mathrm{O}_{4}$ & $\mathrm{C} 15532$ & $\mathrm{HRMS}_{\mathrm{PL}}^{1}$ & 216.099 & 594.1 & 5.049 \\
3838 & N-Acetyl-ornithine & $\mathrm{C}_{7} \mathrm{H}_{14} \mathrm{~N}_{2} \mathrm{O}_{3}$ & $\mathrm{C} 00437$ & $\mathrm{HRMS}_{\mathrm{a}}^{1} \mathrm{R}_{\mathrm{ta}}$ & 173.0931 & 791.2 & 1.068 \\
& Arginine & $\mathrm{C}_{6} \mathrm{H}_{14} \mathrm{~N}_{4} \mathrm{O}_{2}$ & $\mathrm{C} 00062$ & $\mathrm{HRMS}_{\mathrm{PL}}^{1}$ & 173.1044 & 1304.1 & 1.3781 \\
\hline
\end{tabular}

${ }^{a}$ See reference Kanehisa and Goto 2000

b See reference Sumner et al. 2014

${ }^{c}$ Retention time

${ }^{\mathrm{d}} \log _{2}$ fold change in expression of metabolite between planktonic and biofilm data sets

e Not available, fold change unable to be formulated as peak intensities did not significantly change between sample sets

${ }^{\mathrm{f}}$ Not available, fold change unable to be formulated as only detected in the planktonic data set and not in the biofilm data set

Through plotting the average peak intensities between replicates of peaks listed in Table 1 (Online Resource 4), demonstrates that the deviation between replicates in the sample group is relatively small, and highlights differences in the fold changes between sample groups. All individual peak intensities are shown in Online Resource 3, raw data.

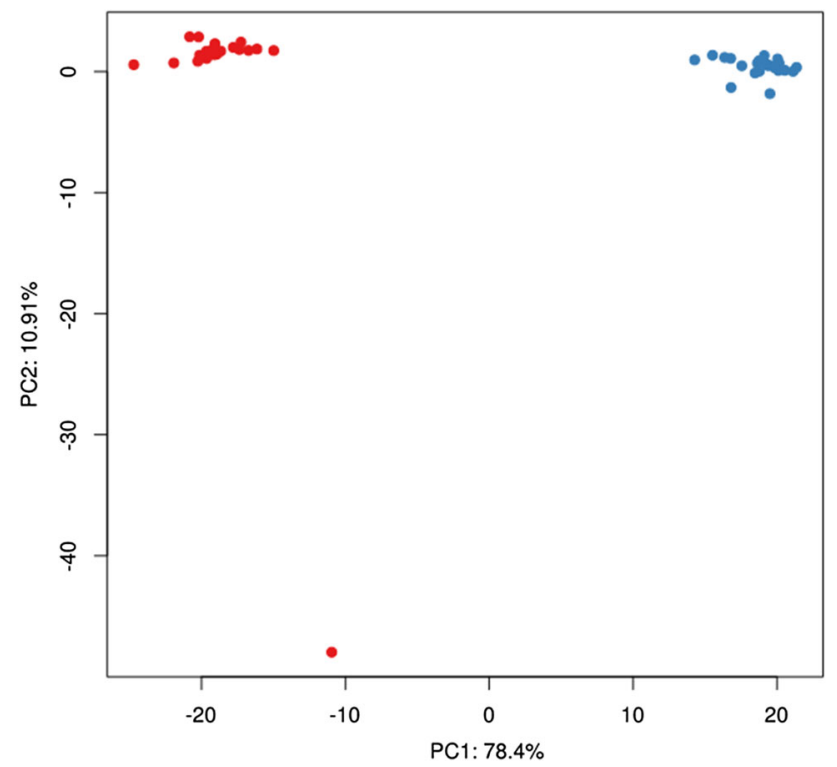

Fig. 2 Principal component analysis (PCA) plots of Planktonic (blue) and biofilm (red) metabolomic data sets, utilising a $10 \mathrm{~min}$ bead beating extraction method; beads in a 50:50 suspension in extraction solvent of chloroform; methanol; water (ratio 1:3:1), followed by liquid-chromatography-mass spectrometry. Red and blue data plots represent planktonic cells and biofilm biological sample replicates, respectively. $\mathrm{n}=24$ (Color figure online)
PCA of metabolomic data sets of planktonic cells and biofilm samples, analysed using LC-MS, revealed clustering of biological replicates (Fig. 2). The plot shows variation in detected metabolites between both groups. Principal components 1 and 2 are responsible for 78.4 and $10.91 \%$ of the variation, respectively. Furthermore, the plot shows an outlier in the biofilm data set not clustered with the other replicates along PCA 2. This sample displays low or zero peak intensities in most metabolites compared to other replicates in the set (see Online Resource 3, raw data, sample B20) and is likely to be due to a failed injection.

\subsection{Arginine Metabolism- an example of a metabolic pathway showing significant changes between planktonic and biofilm samples}

Metabolites were matched to pre-existing pathways defined by the KEGG database (Kanehisa and Goto 2000). The results yielded 129 pathways containing a minimum of two identified $\left(\mathrm{HRMS}_{\mathrm{a}}^{1} \mathrm{R}_{\mathrm{ta}}\right.$ ) or annotated (HRMS $\left.\mathrm{PL}^{1}\right)$ metabolites that demonstrated significant $(\mathrm{P}<0.05)$ changes in expression between planktonic cells and biofilm samples. Table 2 lists the top 20 of these pathways annotated with the most detected metabolites. All further identified and annotated compounds grouped into pathways are listed in Online Resource 3.

After mapping the data to arginine biosynthesis (KEGG pathway 00220), with filtering based on the available genome sequence of this isolate, it was found that 4 identified $\left(\mathrm{HRMS}_{\mathrm{a}}^{1} \mathrm{R}_{\mathrm{ta}}\right)$ and 5 putatively annotated 
Table 2 Top 20 metabolic pathways that have intermediate and end-product metabolites that display significant changes in intensity between planktonic cells and biofilm samples

\begin{tabular}{|c|c|c|c|c|c|c|}
\hline Pathway name $^{\mathrm{a}}$ & $\begin{array}{l}\text { KEGG map } \\
\text { ID }^{\text {a }}\end{array}$ & $\begin{array}{l}\text { Number of } \\
\text { metabolites }\end{array}$ & $\begin{array}{l}\text { Annotated } \\
\left(\mathrm{HRMS}_{\mathrm{PL}}^{1}\right)^{\mathrm{c}}\end{array}$ & $\begin{array}{l}\text { Identified } \\
\left(\mathrm{HRMS}_{\mathrm{a}}^{1} \mathrm{R}_{\mathrm{ta}}\right)^{\mathrm{d}}\end{array}$ & $\begin{array}{l}\text { Coverage } \mathrm{e}^{\mathrm{e}} \\
(\%)\end{array}$ & $\begin{array}{l}P \text { vs } \\
B^{f}\end{array}$ \\
\hline Arginine and proline metabolism/arginine biosynthesis & $00330 / 00220$ & 90 & 49 & 5 & 60 & 33 \\
\hline Protein digestion and absorption & 04974 & 47 & 22 & 7 & 61.7 & 19 \\
\hline Tyrosine metabolism & 00350 & 76 & 35 & 2 & 48.7 & 18 \\
\hline Histidine metabolism & 00340 & 45 & 25 & 3 & 62.2 & 17 \\
\hline Galactose metabolism & 00052 & 41 & 22 & 0 & 53.7 & 17 \\
\hline Aminoacyl-tRNA biosynthesis & 00970 & 52 & 13 & 7 & 38.5 & 17 \\
\hline Cyanoamino acid metabolism & 00460 & 46 & 31 & 1 & 69.6 & 16 \\
\hline Linoleic acid metabolism & 00591 & 28 & 26 & 0 & 92.9 & 15 \\
\hline Limonene and pinene degradation & 00903 & 64 & 53 & 0 & 82.8 & 15 \\
\hline C5-Branched dibasic acid metabolism & 00660 & 32 & 20 & 4 & 75 & 14 \\
\hline Mineral absorption & 04978 & 29 & 11 & 4 & 51.7 & 14 \\
\hline Phosphotransferase system (PTS) & 02060 & 48 & 20 & 1 & 43.8 & 14 \\
\hline Two-component system & 02020 & 41 & 10 & 6 & 39 & 14 \\
\hline Fructose and mannose metabolism & 00051 & 51 & 16 & 0 & 31.4 & 14 \\
\hline Alanine, aspartate and glutamate metabolism & 00250 & 24 & 15 & 2 & 70.8 & 13 \\
\hline Lysine degradation & 00310 & 47 & 26 & 1 & 57.4 & 13 \\
\hline Glycine, serine and threonine metabolism & 00260 & 51 & 27 & 2 & 56.9 & 13 \\
\hline Phenylalanine metabolism & 00360 & 72 & 33 & 1 & 47.2 & 13 \\
\hline Pyrimidine metabolism & 00240 & 66 & 28 & 5 & 50 & 12 \\
\hline Aminobenzoate degradation & 00627 & 84 & 28 & 1 & 34.5 & 12 \\
\hline Purine metabolism & 00230 & 92 & 16 & 9 & 27.2 & 12 \\
\hline Citrate cycle (TCA cycle) & 00020 & 20 & 5 & 3 & 40 & 4 \\
\hline
\end{tabular}

${ }^{a}$ In accordance with KEGG (Kyoto Encyclopaedia of Genes and Genomes data base) (Kanehisa and Goto 2000)

b Total number of metabolites in the pathway according to KEGG

c Number of annotated metabolites detected, metabolite code HRMS ${ }_{\mathrm{PL}}^{1}$ (Sumner et al. 2014)

${ }^{\mathrm{d}}$ Number of identified metabolites detected, metabolite code $\mathrm{HRMS}_{\mathrm{a}}^{1} \mathrm{R}_{\mathrm{ta}}$ (Sumner et al. 2014)

e Percentage of metabolites detected in the pathway $\left(\mathrm{HRMS}_{\mathrm{PL}}^{1}\right.$ and $\mathrm{HRMS}_{\mathrm{a}}^{1} \mathrm{R}_{\mathrm{ta}}$ )

${ }^{\mathrm{f}}$ Number of metabolites detected $\left(\mathrm{HRMS}_{\mathrm{PL}}^{1}\right.$ and $\left.\mathrm{HRMS}_{\mathrm{a}}^{1} \mathrm{R}_{\mathrm{ta}}\right)$ that show significant changes in expression profiles between Planktonic $(\mathrm{P})$ and biofilm (B) samples

$\left(\mathrm{HRMS}_{\mathrm{PL}}^{1}\right.$ ) (total of 9) metabolites are involved in this pathway in S. aureus (Table 1 and Fig. 3). Of these, 6 metabolites ( 2 identified and 4 annotated) were seen to have significant $(\mathrm{P}<0.05) \log _{2}$ fold changes between planktonic and biofilm sample sets. Peak annotation results of the 9 identified or annotated arginine biosynthesis metabolites detected in negative mode are listed in Table 1. A number of metabolites were identified or annotated in both data sets were a fold-change difference in intensity could be calculated (Table 1), however L-arginosuccinate was detected in the planktonic data set, displaying a peak in all sample replicates, but not detected in the biofilm data set. Glutamine was identified in both planktonic and biofilm data sets, however its peak intensities did not significantly change between sample sets. Peak intensity graphs for the peaks listed in Table 1 are shown in Online Resource 5.

\section{Discussion}

Most bacteria possess the ability to live in suspension or as an adhered community on a surface. Novel ways to study the distinct differences in metabolism between these two states are much needed so that differences can be exploited with the development of new antibiotics, in addition to understanding fundamental biological processes. Further to this, studying Gram-positive bacteria and their intracellular metabolic intermediates comes with additional complications due to the robustness of the thick peptidoglycan cell wall. Data presented herein describes a novel metabolite extraction method utilising a direct bead beating method for biofilms cultivated in a 96-well format, and downstream analysis to compare planktonic and biofilm cells. Many previously published approaches for biofilm investigations use 96-well plates, allowing the bacterial culture 
to adhere and develop on the bottom of the well (O'Toole 2011; Merritt et al. 2005; Christensen et al. 1985). The use of a 96-well plate format has the additional benefit of allowing for the substantial multiplexing of samples on the same plate, as previously shown (Pierce et al. 2008; LopezRibot 2014; Srinivasan et al. 2013), including metabolite analysis. Of note is that current published metabolite extraction methods using bead beating focus on bacterial cells living in a planktonic state (Liebeke et al. 2012). This method has previously been shown to be beneficial in the extraction of DNA and metabolites from Gram-positive bacteria (Liebeke et al. 2012), pathogenic yeasts (Bolano et al. 2001) and filamentous fungi (van Burik et al. 1998); organisms that also possess a thick cell wall.

We were unable to use standard enzymatic disruption methods (Salazar 2007), as this would introduce the requirement for ambient temperature resulting in metabolite degradation. However, we compared lysis using bead beating with two other methods, filtration followed by solvent extraction alone (2002; 2003), and sonication (Takahashi et al. 2010). Solvent extraction was demonstrated to be less effective than either bead beating or sonication, while sonication was comparable to bead beating, giving broadly similar results, so can be considered equally viable for analysis of planktonic Gram-positive bacteria. The limitation of sonication for biofilm experiments include the complexities of sonicating uniformly across a multiwell plate and the time taken for bath sonication methods.

Previous metabolomic literature has reported a number of different solvent mixtures, demonstrating that the solvent used and the temperature of extraction determines the diversity of extracted metabolites (A et al. 2005). We used chloroform methanol water 1:3:1 as an optimal extraction solvent for our chromatography system ( $t$ 'Kindt et al. 2010). A practical concern with using solvents such as chloroform in cell-culture plastics is they can etch and degrade plastic (Bawn and Wajid 1956). To avoid this, glass coated wells and glass cell culture materials can be used. However, previous work has demonstrated that biofilm formation is altered on different substrates (Jansen and Kohnen 1995; Ramage et al. 2003; Passerini de Rossi et al. 2007), so the use of plastic is often unavoidable. It was found here that the effects of plastic degradation on overall metabolomic results was minimal, possibly due to the poor retention of hydrophobic compounds on the pHILIC column used, and if present could be compensated for with the use of an appropriate negative control. In addition, any plastic debris would also be pelleted along with the beads at the end of the extraction process, and may be discarded as the supernatant contains the metabolite extract.

One metabolic pathway that shows significant changes is arginine metabolism, specifically the urea cycle (Fig. 3).
Several pathway intermediates: glutamate $\left(\mathrm{HRMS}_{\mathrm{a}}^{1} \mathrm{R}_{\mathrm{ta}}\right)$; $\mathrm{N}$-acetyl-L-glutamate $\left(\mathrm{HRMS}_{\mathrm{a}}^{1} \mathrm{R}_{\mathrm{ta}}\right)$; N-acetyl-L-citrulline $\left(\mathrm{HRMS}_{\mathrm{PL}}^{1}\right)$; aspartate $\left(\mathrm{HRMS}_{\mathrm{PL}}^{1}\right)$; citrulline $\left(\mathrm{HRMS}_{\mathrm{a}}^{1} \mathrm{R}_{\mathrm{ta}}\right)$; arginine $\left(\mathrm{HRMS}_{\mathrm{PL}}^{1}\right)$; and N-acetyl-ornithine $\left(\mathrm{HRMS}_{\mathrm{a}}^{1} \mathrm{R}_{\mathrm{ta}}\right)$ were detected to be significantly up-regulated in planktonic samples or down-regulated in the biofilm samples, suggesting that they are depleted in response to rapid flux through this pathway. These results correlate with previous studies that show significant changes in energy and cell metabolism between planktonic cells and biofilms (Zhang and Powers 2012; Ammons et al. 2014; Gjersing et al. 2007). Previous work using microarrays also showed upregulation of urea cycle genes in response to the formation of biofilms in S. aureus (Resch et al. 2005). The amino acid glutamine was identified (HRMS $S_{\mathrm{a}}^{1} \mathrm{R}_{\mathrm{ta}}$ ) in both data sets but was seen not to be changing in intensity between planktonic and biofilm samples. $S$. aureus selectively extracts the amino acid glutamine from its medium environment (Zhu et al. 2007). The metabolite L-arginosuccinate was annotated $\left(\mathrm{HRMS}_{\mathrm{PL}}^{1}\right.$ ) to be found in planktonic samples only and was absent from biofilm data. The absence in biofilms may be because the metabolite is simply not present or because its concentration is below the limits of detection of our instrumentation. Previous studies by Zhu et al. (2007) and Ammons et al. (2014) suggest that changes in amino acid metabolism are a key feature differing in biofilms compared to planktonic samples. Furthermore, these studies and $\mathrm{Wu}$ et al. (2010) suggest that arginine metabolism and catabolism play an important role in biofilm survival. We hypothesise that $S$. aureus may amplify flux through the urea cycle to generate ammonia to restore $\mathrm{pH}$ balance in response to the production of acid in the biofilm ECM (Resch et al. 2005) due to anaerobic glycolysis. This correlates with other studies that suggest that amino acid catabolism is crucial for biofilm $\mathrm{pH}$ balance (Beenken et al. 2004; Resch et al. 2006; Resch et al. 2005; Zhu et al. 2007).

This study highlights key metabolic differences in arginine metabolism between different bacterial growth states. Findings presented here show that metabolism is significantly altered by the same species of bacteria once a sessile growth phase has been initiated. The changes identified here between growth states could be evidence of the bacteria responding to their changing environment and trying to maintain a 'status quo' in chemical and $\mathrm{pH}$ balance, Bacteria should be thought of as dynamic entities capable of displaying significantly altered phenotypes without the necessity of genetic change. Because of this, research into bacteria, and especially in the field of antimicrobial testing and identification, should consider different effects drugs may have on different stages of growth. 


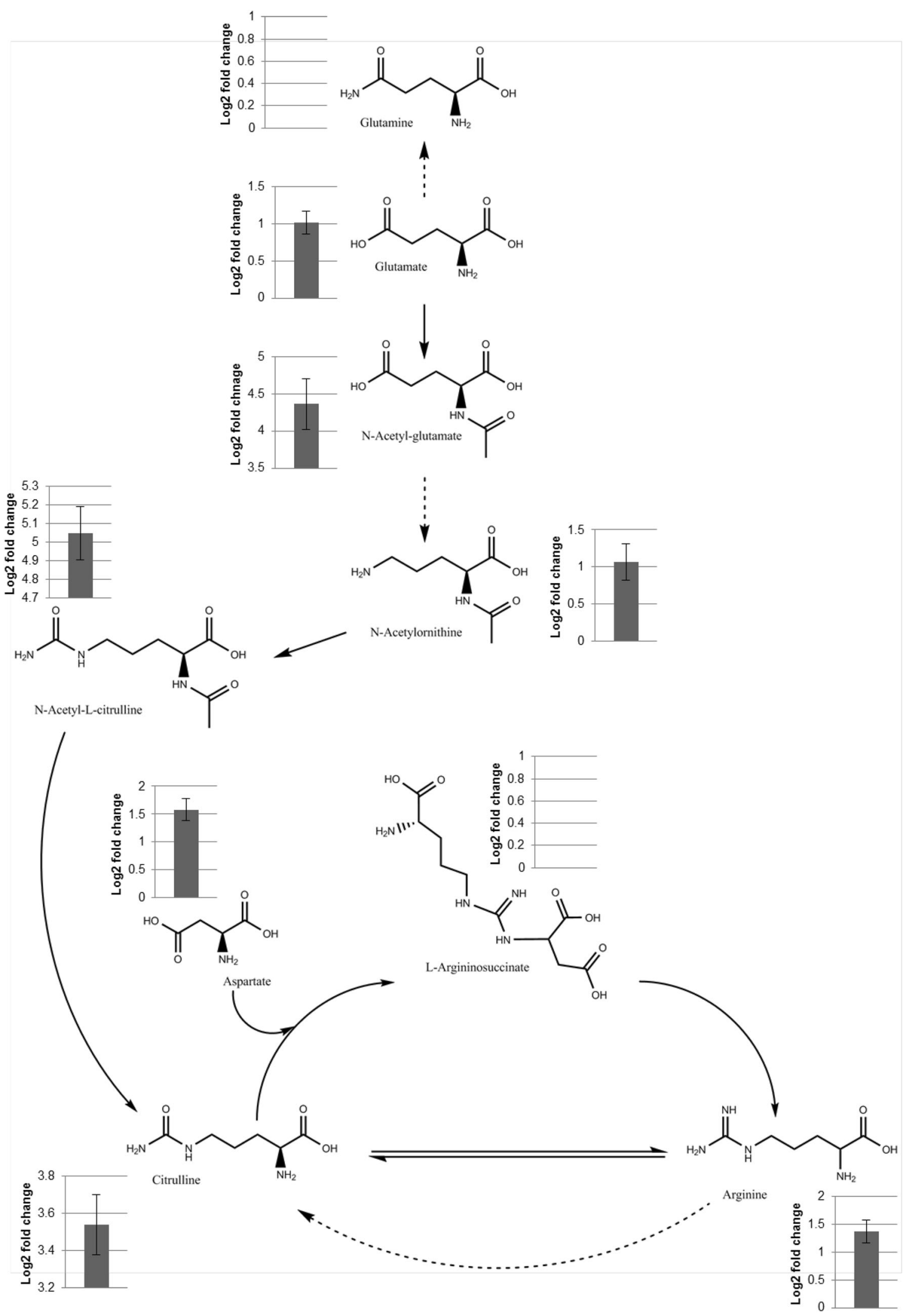


4Fig. 3 Arginine biosynthesis metabolic pathway showing both detected metabolites identified by having mass and retention time $\left(\mathrm{R}_{\mathrm{t}}\right)$ matched to an internal standard (Bold and underlined) and annotated metabolites matched to an accurate mass database entry. Full molecular structures of the metabolites are given. Graphs represent $\log _{2}$ fold change of metabolite intensity/expression in planktonic cells compared to biofilms. A positive fold change indicates expression was either up regulated in planktonic samples or down regulated in biofilm samples. Pathway and structures annotated from the Kyoto Encyclopedia of Genes and Genomes (KEGG) database. Block arrows represent a direct link between metabolites. Dashed arrows represent the presence of metabolite intermediates that were not detected. Error bars represent $99.95 \%$ fold change confidence interval

\subsection{Method limitations}

The biofilm cultivation method presented here in a 96-well format, is based on a static growing biofilm. It is known that biofilm phenotype can change depending on whether cultivated in a static or flow system (Weaver et al. 2012; Yarwood et al. 2004). However, the bead-beating extraction method described here can be applied to biofilms cultivated on cover-slips used in a flow system, whereby the cover-slip with attached biofilm is removed, washed and placed in a bijou or similar for extraction. The extraction method also does not discriminate between the biofilm encased cells and the ECM. An appropriate ECM and cell extraction method, suitable for mass-spectrometry metabolomic samples is a target for future method development. Finally, due to the resilience of the cells and the time taken for full lysis and extraction, rapid metabolic processes may still continue under these conditions, and should be taken into account when evaluating the results.

\section{Conclusions}

The low-temperature, rapid, mechanical method coupled with an extraction solvent to lyse the peptidoglycan cell wall and extract the cellular metabolome from Gram-positive cells living in a planktonic state or as a biofilm is described here. The method is shown to be a highly reproducible platform to study cellular metabolism. PCA clustering of 24 replicates, low variation in average peak intensities and identification of significant (adjusted $\mathrm{P}<0.05)$ changes in abundance of metabolite components of the arginine biosynthesis pathway between data sets highlights this reproducibility. Comparison with other methods for lysis of planktonic bacteria further demonstrates its utility for general bacterial metabolomics.

Data shows that significant changes in a number of metabolic pathways, highlighting arginine biosynthesis, take place between planktonic cells and biofilms. During static culture, the biofilm's adherence to the base of the well will necessarily result in reduced access to oxygen, in contrast with a planktonic, shaking culture, and this change in oxygen availability may be responsible for some or many of the changes observed. However, is a general consensus that metabolism is significantly altered in biofilm-forming cells compared to planktonic cells (Zhang and Powers 2012; Resch et al. 2006; Gjersing et al. 2007). The study of microbial metabolism is challenging but provides crucial insight into the biochemistry of bacteria. The added complication of bacterial physiology responding to its environment adds to the complexity of such studies. The ability to study metabolism provides the potential for inferences to be gained, such as the modes of action of antimicrobials and the identification of new metabolic drug targets. The insurgence of antimicrobial resistance (Arias and Murray 2015) means that research in this area is vitally important.

Acknowledgments We would like thank Dr Stefan Weidt of Glasgow Polyomics, for help with running MS samples and data interpretation. We would like to thank Prof. Michael P. Barrett of Glasgow Polyomics and the University of Glasgow for comments on the manuscript. We would also like to thank Margaret Mullin of the University of Glasgow for her help with the processing of samples for scanning electron microscopy.

Author contributions LHS carried out the work and drafted the manuscript. MJD and RLD provided help and supervision with the project and initial experimental work study design. FM provided bioinformatics support and participated in data analysis. GR participated in study design, data analysis and manuscript writing. KEVB provided overall supervision of project, financial support, study design and manuscript drafting. Authors read and approved final manuscript prior to submission.

Financial Support Scottish Funding Council provided funding for the purchasing of the instruments, including the mass-spectrometers and liquid chromatography systems. Glasgow Polyomics is supported by the University of Glasgow and Wellcome Trust (Grant numbers 097821/Z/11/Z and 105614/Z/14/Z). This work was supported by the Doctoral Training Centre (DTC) (Grant number EP/F500424/1) in Technologies at the Interface between Engineering, the Physical Sciences and the Life Sciences, University of Glasgow. The DTC is funded by the Engineering and Physical Sciences Research Council (EPSRC) and the Biotechnology and Biological Sciences Research Council (BBSRC).

\section{Compliance with ethical standards}

Conflicts of Interest The authors declare no conflict of interest.

Research Involving Human Participants and/or Animals No studies contained in this article involved human participants or animals. Informed consents were not required for this study.

Open Access This article is distributed under the terms of the Creative Commons Attribution 4.0 International License (http://crea tivecommons.org/licenses/by/4.0/), which permits unrestricted use, distribution, and reproduction in any medium, provided you give appropriate credit to the original author(s) and the source, provide a link to the Creative Commons license, and indicate if changes were made. 


\section{References}

A, J. et al., 2005. Extraction and GC/MS analysis of the human blood plasma metabolome. Analytical Chemistry, 77(24), 8086-8094. Available at: http://www.ncbi.nlm.nih.gov/pubmed/16351159.

Ammons, M. C. B., et al. (2014). Quantitative NMR metabolite profiling of methicillin-resistant and methicillin-susceptible Staphylococcus aureus discriminates between biofilm and planktonic phenotypes. Journal of Proteome Research, 13, 2973-2985.

Arias, C. A., \& Murray, B. E. (2015). A new antibiotic and the evolution of resistance. New England Journal of Medicine, 372(12), 1168-1170. doi:10.1056/NEJMcibr1500292.

Bawn, C. E. H., \& Wajid, M. A. (1956). High polymer solutions. Part 7- Vapour pressure of polystyrene solutions in acetone, chloroform and propyl acetate. Transactions of the Faraday Society, $52,1658-1664$.

Beenken, K.E. et al., 2004. Global gene expression in Staphylococcus aureus biofilms. Journal of bacteriology, 186(14), 4665-4684. Available at: http://www.pubmedcentral.nih.gov/articlerender. fcgi artid $=438561 \&$ tool $=$ pmcentre $\&$ rendertype $=$ abstract .

Bolano, A. et al., 2001. Rapid methods to extract DNA and RNA from Cryptococcus neoformans. FEMS Yeast Research, 1(3), 221-4. Available at: http://www.ncbi.nlm.nih.gov/pubmed/12702347.

Bolstad, B.M. et al., 2003. A comparison of normalization methods for high density oligonucleotide array data based on variance and bias. Bioinformatics (Oxford, England), 19(2), 185-193.

Bolten, C.J. et al., 2007. Sampling for metabolome analysis of microorganisms. Analytical Chemistry, 79(10), 3843-3849. Available at: http://www.ncbi.nlm.nih.gov/pubmed/17411014.

Cassou, C. A., et al. (2014). Electrothermal supercharging in mass spectrometry and tandem mass spectrometry of native proteins. Analytical Chemistry, 85(1), 138-146.

Christensen, G., et al. (1985). Adherence of coagulase-negative staphylococci to plastic tissue culture plates: A staphylococci to medical devices. Adherence of coagulase-negative staphylococci to plastic tissue culture plates: A quantitative model for the adherence of staphylococci. Journal of Clinical Microbiology, 22(6), 996-1006.

Creek, D.J. et al., 2012. IDEOM: An Excel interface for analysis of LC-MS-based metabolomics data. Bioinformatics (Oxford, England), 28(7), 1048-1049.

Creek, D. J., et al. (2011). Identification by retention time prediction. Bioinformatics Application Note, 28(7), 8703-8710.

de Koning, W. \& van Dam, K., 1992. A method for the determination of changes of glycolytic metabolites in yeast on a subsecond time scale using extraction at neutral $\mathrm{pH}$. Analytical biochemistry, 204(1), 118-123. Available at: http://www.ncbi.nlm.nih. gov/pubmed/1514678.

Drenkard, E. \& Ausubel, F.M., 2002. Pseudomonas biofilm formation and antibiotic resistance are linked to phenotypic variation. Nature, 416(6882), 740-743. Available at: http://www.ncbi.nlm. nih.gov/pubmed/11961556.

Erlandsen, S.L. et al., 2004. High-resolution visualization of the microbial glycocalyx with low-voltage scanning electron microscopy: dependence on cationic dyes. The Journal of Histochemistry and Cytochemistry: Official Journal of the Histochemistry Society, 52(11), 1427-1435. Available at: http://www.pubmed central.nih.gov/articlerender.fcgi?artid=3957825\&tool=pmcentrez \&rendertype=abstract. Accessed September 25, 2014.

Faijes, M., Mars, A.E. \& Smid, E.J., 2007. Comparison of quenching and extraction methodologies for metabolome analysis of Lactobacillus plantarum. Microbial cell factories, 6, 27. Available at: http://www.pubmedcentral.nih.gov/articlerender. fcgi?artid=2031893\&tool=pmcentrez\&rendertype=abstract. Accessed September 12, 2014.

Fiehn, O., 2002. Metabolomics-the link between genotypes and phenotypes. Plant Molecular Biology, 48(1-2), 155-171. Available at: http://www.ncbi.nlm.nih.gov/pubmed/11860207.

Gjersing, E.L. et al., 2007. NMR metabolomics of planktonic and biofilm modes of growth in Pseudomonas aeruginosa. Analytical chemistry, 79(21), 8037-8045. Available at: http://www.ncbi. nlm.nih.gov/pubmed/17915964.

Gross, J.H., 2011. Mass spectrometry a textbook (2nd Edn). In J. H. Gross (Ed.), Analytical Chemistry. Springer

Hedges, J. B., et al. (2013). Effects of ammonium bicarbonate on the electrospray mass spectra of proteins: evidence for bubbleinduced unfolding. Analytical Chemistry, 85, 6469-6476.

Hochberg, Y. \& Benjamini, Y., 1990. More powerful procedures for multiple significance testing. Statistics in medicine, 9(7), 811-818. Available at: http://www.ncbi.nlm.nih.gov/pubmed/ 2218183.

Horning, E.C. \& Horning, M.G., 1971. Metabolic profiles: gas-phase methods for analysis of metabolites. Clinical chemistry, 17(8), 802-829. Available at: http://www.ncbi.nlm.nih.gov/pubmed/ 5105517.

Jansen, B. \& Kohnen, W., 1995. Prevention of biofilm formation by polymer modification. Journal of industrial microbiology, 15(4), 391-396. Available at: http://www.ncbi.nlm.nih.gov/pubmed/ 8605077.

Kanehisa, M. \& Goto, S., 2000. KEGG: kyoto encyclopedia of genes and genomes. Nucleic acids research, 28(1), 27-30. Available at: http://www.pubmedcentral.nih.gov/articlerender.fcgi?artid= $102409 \&$ tool $=$ pmcentrez\&rendertype $=$ abstract.

Laidler, K., 1984. The development of the Arrhenius equation. Journal of Chemical Education, 61(6), 494. Available at: http:// pubs.acs.org/doi/abs/10.1021/ed061p494. Accessed July 31, 2014.

Liebeke, M. et al., 2012. Metabolome analysis of gram-positive bacteria such as Staphylococcus aureus by GC-MS and LC-MS. Methods in molecular biology (Clifton, N.J.) (Vol. 815, pp. 377-398).

Lopez-Ribot, J. L., 2014. Large-scale biochemical profiling of the candida albicans biofilm matrix: New compositional, structural, and functional insights. mBio, 5(5).

Maharjan, R.P. \& Ferenci, T., 2003. Global metabolite analysis: The influence of extraction methodology on metabolome profiles of Escherichia coli. Analytical biochemistry, 313(1), 145-154. Available at: http://www.ncbi.nlm.nih.gov/pubmed/12576070.

Merritt, J., Kadouri, D. \& O'toole, G., 2005. Growing and analyzing static biofilms. Current protocols in ..., Chapter 1(Unit 1B.1). Available at: http://onlinelibrary.wiley.com/doi/10.1002/ 9780471729259.mc01b01s22/full. Accessed July 31, 2014.

Nicholson, J. K., \& Lindon, J. C. (2008). Systems biology: Metabonomics. Nature, News and Views Q\&A, 455, 1054-1056.

O'Toole, G.A., 2011. Microtiter Dish Biofilm Formation Assay. Journal of Visualized Experiments: JoVE, (47), 2437. Available at: http://www.ncbi.nlm.nih.gov/pmc/articles/PMC3182663/.

O’Toole, G., Kaplan, H. B., \& Kolter, R. (2000). Biofilm formation as microbial development. Annual Review of Microbiology, 54, 49-79.

Oliver, S.G. et al., 1998. Systematic functional analysis of the yeast genome. Trends in biotechnology, 16(9), 373-378. Available at: http://www.ncbi.nlm.nih.gov/pubmed/9744112.

Passerini de Rossi, B. et al. 2007. Biofilm formation by Stenotrophomonas maltophilia isolates from device-associated nosocomial infections. Revista Argentina de microbiología, 39(4), 204-212. Available at: http://www.ncbi.nlm.nih.gov/pubmed/ 18390153 
Periasamy, S. et al., 2012. How Staphylococcus aureus biofilms develop their characteristic structure. Proceedings of the National Academy of Sciences of the United States of America, 109(4), 1281-1286. Available at: http://www.pubmedcentral.nih. gov/articlerender.fcgi? artid=3268330\&tool=pmcentrez\&render type=abstract. Accessed September 26, 2014.

Pierce, C.G. et al., 2008. A simple and reproducible 96-well platebased method for the formation of fungal biofilms and its application to antifungal susceptibility testing. Nature Protocols, 3(9), 1494-1500. Available at: http://www.nature.com/doifinder/ 10.1038/nprot.2008.141. Accessed September 13, 2014.

Ramage, G. et al. 2003. Formation of Propionibacterium acnes biofilms on orthopaedic biomaterials and their susceptibility to antimicrobials. Biomaterials, 24(19), 3221-3227. Available at: http://linkinghub.elsevier.com/retrieve/pii/S014296120300173X. Accessed July 31, 2014.

Resch, A. et al. 2006. Comparative proteome analysis of Staphylococcus aureus biofilm and planktonic cells and correlation with transcriptome profiling. Proteomics, 6(6), 1867-1877. Available at: http://www.ncbi.nlm.nih.gov/pubmed/16470655. Accessed May 22, 2014.

Resch, A., et al. (2005). Differential gene expression profiling of Staphylococcus aureus cultivated under biofilm and planktonic conditions differential gene expression profiling of staphylococcus aureus cultivated under biofilm and planktonic conditions. Applied and Environmental Microbiology, 71(5), 2663-2676.

Salazar, O. \& Asenjo, J. A, 2007. Enzymatic lysis of microbial cells. Biotechnology Letters, 29(7), 985-994. Available at: http:// www.ncbi.nlm.nih.gov/pubmed/17464453. Accessed July 10, 2014.

Scheltema, R. A et al. 2011. PeakML/mzMatch: a file format, Java library, R library, and tool-chain for mass spectrometry data analysis. Analytical chemistry, 83(7), 2786-2793. Available at: http://www.ncbi.nlm.nih.gov/pubmed/21401061.

Scientific Industries, Inc, 2014. Cell Disruptor. Cell Disrupter Disrupter Genie Literature. Available at: http://www.scientificindustries. com/pdf/disruptorgenie-literature.pdf. Accessed September 18, 2014.

Smith, C. A et al., 2006. XCMS: Processing mass spectrometry data for metabolite profiling using nonlinear peak alignment, matching, and identification. Analytical chemistry, 78(3), 779-787. Available at: http://www.ncbi.nlm.nih.gov/pubmed/16448051.

Smith, K. et al., 2008. Biofilm formation by Scottish clinical isolates of Staphylococcus aureus. Journal of medical microbiology, 57(8), 1018-23. Available at: http://www.ncbi.nlm.nih.gov/ pubmed/18628505. Accessed July 17, 2014.

Smyth, G.K., 2004. Linear models and empirical bayes methods for assessing differential expression in microarray experiments. Statistical applications in genetics and molecular biology, 3(1), p.Article3. Available at: http://www.ncbi.nlm.nih.gov/pubmed/ 16646809. Accessed July 9, 2014.

Soga, T., et al. (2002). Simultaneous determination of anionic intermediates for Bacillus subtilis metabolic pathways by capillary electrophoresis electrospray ionization mass spectrometry. Analytical Chemistry, 74(10), 2233-2239. doi:10.1021/ ac020064n.

Soga, T., et al. (2003). Quantitative metabolome analysis using capillary electrophoresis mass spectrometry. Journal of Proteome Research, 2(5), 488-494. doi:10.1021/pr034020m.

Srinivasan, A. et al., 2013. High-throughput nano-biofilm microarray for antifungal drug discovery. mBio, 4(4). Available at: http:// www.pubmedcentral.nih.gov/articlerender.fcgi?artid=3697808\& tool $=$ pmcentre $\&$ rendertype $=$ abstract.

Stipetic, L. H., et al. (2015). Draft genome sequence of isolate Staphylococcus aureus LHSKB clinical, isolated from an infected hip. Genome announcements, 3(2), e00336.

Sumner, L., et al. (2014). Proposed quantitative and alphanumeric metabolite identification metrics. Metabolomics, 10(6), 1047-1049. doi:10.1007/s11306-014-0739-6.

t'Kindt, R. et al., 2010. Towards an unbiased metabolic profiling of protozoan parasites: Optimisation of a Leishmania sampling protocol for HILIC-orbitrap analysis. Analytical and Bioanalytical Chemistry, 398(5), 2059-2069. Available at: 10.1007/ s00216-010-4139-0.

Takahashi, N., Washio, J. \& Mayanagi, G., 2010. Metabolomics of supragingival plaque and oral bacteria. Journal of Dental Research, 89(12), 1383-1388. Available at: http://www.ncbi. nlm.nih.gov/pubmed/20924070. Accessed July 19, 2014.

Tang, J., 2011. Microbial metabolomics. Current genomics, 12(6), 391-403. Available at: http://www.pubmedcentral.nih.gov/ articlerender.fcgi?artid $=3178908 \&$ tool=pmcentrez $\&$ rendertype $=$ abstract.

van Burik, J.-A. H., et al. (1998). Comparison of six extraction techniques for isolation of DNA from filamentous fungi. Medical Mycology, 36(5), 299-303.

Weaver, W. M. et al., 2012. Fluid flow induces biofilm formation in Staphylococcus epidermidis polysaccharide intracellular adhesin-positive clinical isolates. Applied and Environmental Microbiology, 78(16), 5890-5896. Available at: http://aem.asm.org/ content/78/16/5890.abstract.

Wu, X.-H., et al. (2010). Sampling methods for NMR-based metabolomics of Staphylococcus aureus. Biotechnology Journal, 5(1), 75-84. doi:10.1002/biot.200900038.

Yarwood, J. M., et al. (2004). Quorum sensing in Staphylococcus aureus biofilms. Journal of Bacteriology, 186(6), 1838-1850.

Zhang, B., \& Powers, R. (2012). Analysis of bacterial biofilms using NMR-based metabolomics. Future Medicinal Chemistry, 4(10), 1273-1306.

Zhu, Y. et al., 2007. Staphylococcus aureus biofilm metabolism and the influence of arginine on polysaccharide intercellular adhesin synthesis, biofilm formation, and pathogenesis. Infection and Immunity, 75(9), 4219-26. Available at: http://www.pubmedcen tral.nih.gov/articlerender.fcgi?artid=1951195\&tool=pmcentrez\& rendertype=abstract. Accessed May 13, 2014. 